\title{
Preoperative visualization of the lingual nerve by 3D double-echo steady-state MRI in surgical third molar extraction treatment
}

\author{
Adib Al-Haj Husain ${ }^{1} \cdot$ Silvio Valdec $^{1} \cdot$ Bernd Stadlinger $^{1} \cdot$ Martin Rücker $^{2} \cdot$ Marco Piccirelli $^{3} \cdot$ Sebastian Winklhofer $^{3}(\mathbb{C}$
}

Received: 29 March 2021 / Accepted: 18 September 2021 / Published online: 29 September 2021

(c) The Author(s) 2021

\begin{abstract}
Objectives To assess the lingual nerve (LN) visualization using a 3D double-echo steady-state MRI sequence (3D-DESS). Materials and methods Three readers prospectively evaluated the LN for its continuous visibility in 3D-DESS MRI in 19 patients with an indication for removal of mandibular impacted third molars, using a 5 -point scale $(4=$ excellent to $0=$ none). Six LN anatomical intermediate points (IP) were selected and checked for their detectability by a 4-point scale $(4=$ yes to1 $=$ no). Inter- and intra-rater agreement was evaluated using intraclass correlation coefficient and percentage of agreement. Results The average nerve continuity score was $3.3 \pm 0.46$. In $35 \%$ of the cases, the entire course was continuously visible. In $10 \%$, the proximal and $60 \%$, the distal part of the nerve was not continuously visible. Inter- and intra-reader agreement was good (ICC $=0.76$, ICC $=0.75$ ). The average detectability score of all IP was $3.7 \pm 0.41$. From IP1 to IP5, the detectability was excellent; meanwhile, IP6 had lower visibility. The inter- and intra-reader percentage of agreement was $77 \%$ and $87 \%$. Conclusions The 3D-DESS sequence allowed accurate and continuous visualization of the LN with high reproducibility in more than one-third of the patients. This could improve the preoperative clarification of the LN position and thereby reduce complications during dentoalveolar surgical interventions.

Clinical relevance 3D-DESS MRI might be beneficial in clinical scenarios where the second molar is elongated or presents a difficult rotational position while simultaneously having a close positional relationship to the third molar. Thereby, osteotomy performed more lingually, indicating extended lingual flap detachment may increase the risk of LN damage.
\end{abstract}

Keywords Anatomy $\cdot$ Lingual nerve $\cdot$ Magnetic resonance imaging $\cdot$ Oral surgery $\cdot$ Radiology

Marco Piccirelli and Sebastian Winklhofer are equal contributors.

Marco Piccirelli

marco.piccirelli@usz.ch

Sebastian Winklhofer

sebastian.winklhofer@usz.ch

1 Clinic of Cranio-Maxillofacial and Oral Surgery, Center of Dental Medicine, University of Zurich, Plattenstrasse 11, 8032, Zurich, Switzerland

2 Clinic of Cranio-Maxillofacial and Oral Surgery, University Hospital of Zurich, University of Zurich, Rämistrasse 100, 8091, Zurich, Switzerland

3 Department of Neuroradiology, Clinical Neuroscience Center, University Hospital Zurich, University of Zurich, Frauenklinikstrasse 10, 8091 Zurich, Switzerland

\section{Introduction}

Knowing the exact anatomical course of the lingual nerve $(\mathrm{LN})$ is important in various dental and oral surgical interventions. The nerve demonstrates large individual anatomical variability regarding position and proximity to important structures, especially in the third molar region.

Iatrogenic injuries of the $\mathrm{LN}$ can occur in various surgical procedures such as osteotomy of mandibular ramus, orthognathic surgery, endodontic treatments, tumor excision, or local anesthetic application prior to extraction of the mandibular third molar and due to the extraction itself. The insertion of dental implants or even general periodontal procedures can also damage the trigeminal nerve's extracranial branch [1].

Injuring the $\mathrm{LN}$, a rare complication during the removal of mandibular third molars [2,3], is associated with sensory disturbances in the ipsilateral anterior two-thirds of the tongue [4]. The taste is affected due to the connection 
with the special sensory taste fibers of the chorda tympani; significantly affected are the fungiform papillae, which represent the largest part of the taste buds and are responsible for the perception of different taste qualities [5, 6]. Besides, it leads to an altered salivary flow rate of the ipsilateral affected sublingual gland [7]. Varying numbers about the frequency of LN damage risk in patients undergoing third molar extraction are given in the literature, ranging from 0.02 to $2 \%$ [8]. Reports in the literature suggest that the incidence of surgically induced postoperative temporary LN damage varied from 0 to $37.5 \%$, with permanent $\mathrm{LN}$ damage estimated at 0 to $2 \%$ [9]. Consequently, most LN injuries recover spontaneously within 8 weeks; meanwhile, longlasting injuries remain permanent and ensure for affected patients' significant restrictions in everyday life and loss of quality of life [10].

Panoramic radiography is obtained routinely before third molar extraction. However, in numerous cases, where the roots have close proximity to the inferior alveolar canal with a darkening of at least one of the roots, diversion of the inferior alveolar canal, or where a discontinuous cortical line of the nerve canal is present, two-dimensional imaging is not satisfactory [11]. In these cases, the use of three-dimensional imaging such as cone beam computed tomography (CBCT) is indicated and currently the method of choice to decrease the risk of nerve damage [11]. These conventional $\mathrm{x}$-ray-based imaging modalities can only display nervous tissue indirectly by depicting the osseous boundaries surrounding the nerve canal, whereas the nerve itself cannot be visualized. Magnetic resonance imaging (MRI), with its high soft tissue contrast, is the gold standard for imaging of nervous tissues directly, while it is limited for hard tissue imaging yet. The problem with MRI in the oral cavity is the susceptibility to movement artifacts, complex anatomical courses, small sizes and variations of several blood vessels and nerves, and artifacts due to field inhomogeneity caused by metallic implants or dental restorations [12,13].

The visualization of the extracranial nerves' complete course is a major challenge although imaging techniques have greatly improved. Available modalities are high-resolution ultrasound examinations, allowing us to identify the LN intraorally [14], and magnetic resonance neurographic orthopantomogram that allows the simultaneous visualization of bone texture and neural microarchitecture of peripheral nerves [15]. The difficulty in MR neurography has been for a long time the selective and continuous imaging of the thinnest peripheral extracranial nerves. The use of high-resolution three-dimensional turbo spin-echo with variable flip angle (SPACE) STIR sequence with its T2-weighted imaging contrast and background suppression allows depicting the morphology and pathology of peripheral nerves continuously and directly [16].
Other studies, investigating the 3D double-echo steadystate MRI (3D-DESS) sequences showed excellent LN identification $[17,18]$ and precise localization of the inferior alveolar nerve within the mandibular canal [19]. "Black Bone" MRI sequences-such as 3D-DESS and 3D STIRshowed good feasibility and excellent visualization of the inferior alveolar nerve and LN in healthy subjects, whereby the 3D SPACE STIR showed superior signal to noise ratio and nerve muscle contrast to noise ratio, but on the other hand, the DESS sequence was best suited for the comparability of quantitative LN parameters [18]. DESS combines the FID signal of FISP with the echo-signal of PSIF, increasing its $\mathrm{T} 2 *$ specificity and decreasing signal dropout through dephasing. In this MRI sequence, the mandibular nerve's peripheral branches, such as the LN, appear as a high signal intensity structure and could be distinguished from adjacent anatomical structures due to the myelin layer surrounding the nerve, allowing excellent visualization by application of the water excitation fat-suppression technique [20]. Fujii et al. focused on the detectability of the medial and distal portion of the LN at the intermediate point (IP) where the LN starts running from laterally to medially, while Burian et al. in 2019 checked the overall LN detectability using multiple MRI sequences. Previously conducted studies had various limitations, such as visualization of the $\mathrm{LN}$ in a cohort of patients without an indication for third molar extraction [17] or by comparing the visibility of the LN in MRI without checking for its continuous detectability explicitly in the surgically relevant third molar region [18].

The aim of this study was to investigate the detectability of the entire continuous course and the focal detectability of the intermediate points of the $\mathrm{LN}$ in patients with impacted third molars. We focused on the anatomical relationship of the third molar teeth, the anatomic proximity to the alveolar ridge of the lingual cortical plate, and the diameter of the $\mathrm{LN}$ using the 3D-DESS sequence.

\section{Materials and methods}

\section{Study design and setup}

Between May 2018 and December 2018, 23 patients were recruited. Four patients did not complete imaging procedures due to not showing up. Therefore, 38 lingual nerves were evaluated (19 patients, each left and right side). The study population included patients with an indication for removal of partially retained, fully retained, or impacted third molar in the third or fourth mandibular quadrant with the additional indication for three-dimensional imaging according to the guidelines of the Swiss association of dentomaxillofacial radiology admitted to the Clinic of Cranio-Maxillofacial and Oral Surgery of the Center of 
Dental Medicine (University of Zurich) either by a private practitioner or by themselves. MRI data acquisitions were performed by trained neuroradiologists. The subsequent surgical procedure of the mandibular third molar under local anesthesia was performed by oral surgeons. The cohort group included six males $(32 \%)$ and 13 females $(68 \%)$; the mean age of the enrolled patients was $30.5 \pm 13$ years (median age, 25 years; age range, 18-63 years) (Table 1).

The following criteria were required for the study participants to be included: (1) indication for removal of mandibular partially retained, fully retained, or impacted third molar; (2) male and female patients aged 18 to 65 years. The exclusion criteria were (1) acute odontogenic infection; (2) nerve damage of the inferior alveolar nerve or trigeminal nerve; (3) adjacent implants or metallic reconstructions; (4) pregnancy; and (5) other contraindications for MRI imaging.

The study (BASEC-Nr. 2017-01053) was approved by the the Cantonal Ethics Commission of Zurich (Switzerland). All participants were informed and provided written informed consent for inclusion in the study in accordance with the Declaration of Helsinki and its later revised ethical standards.

Reporting complies with the STROBE ("Strengthening the Reporting of Observational studies in Epidemiology") guidelines.

Table 1 Patient characteristics

\begin{tabular}{ll}
\hline Patient characteristics & Total \\
\hline$N$ & 19 \\
Gender, male/female, $N$ & $6 / 13$ \\
Mean (SD) age at scan, years & $30.5(13)$ \\
Median age at scan, years & 25 \\
Age range, years & $18-63$ \\
Totally evaluated lingual nerves & 38 \\
Clinical indication & Third \\
& molar \\
& extrac- \\
Retention types [21] & tion \\
Type $1, N$ & \\
Type $2, N$ & 0 \\
Type $3, N$ & 0 \\
Type $4, N$ & 11 \\
Type $5, N$ & 19 \\
Type 6, $N$ & 2 \\
Type $7, N$ & 0 \\
No retention & 0 \\
Absent & 4 \\
\hline
\end{tabular}

\section{MRI data acquisition}

The MR images were acquired with a 3-Tesla Skyra (release VE11c, Siemens Healthineers, Erlangen, Germany) and a Siemens standard 64-channel head-and-neck coil. The used axial 3D-DESS with water excitation MRI sequence had an isotropic acquisition resolution of $0.75 \times 0.75 \times 0.75$ $\mathrm{mm}^{3} \mathrm{~mm}$ together with a receive bandwidth of $355 \mathrm{~Hz} /$ Px. The other sequence parameters were field-of-view $242 \times 242 \times 78 \mathrm{~mm}^{3}$; acquisition matrix $320 \times 320 \times 104$; slice oversampling $100 \%$; no parallel acquisition; one signal average; acquisition time 12:24 min:s; TR/TE1/TE2 11.2/4.2/7.7 ms; flip angle $30^{\circ}$; and selective water excitation (Table 2).

\section{Image analysis}

The MRI data was stored and evaluated in syngo.via (release VB30a, Siemens Healthineers, Erlangen, Germany) using a 2-MP high-resolution liquid-crystal display. The entire course of the LN was evaluated in randomized patient order by three readers with varying degrees of experience (reader A, attending board-certified neuroradiologist; reader B, attending board-certified oral surgeon; reader $\mathrm{C}$, fourth-year dental medicine student). Before performing the evaluation, all three readers conducted a calibration session, in which some random cases were evaluated together to resolve any uncertainty. Intra-reader agreement was examined by having all readers repeat the readout after a time interval of minimum 2 weeks to avoid recall bias. The intra-reader agreement of reader $\mathrm{C}$ was selected to determine the expected lower bound for that particular non-neuroradiologist expert and investigate its applicability in the daily dental routine. All readers were blinded to the results of the other readers or their previous readout. Axial, coronal, and sagittal DESS multiplanar image reconstructions were available for

Table 2 Main technical parameters of the 3D-DESS MRI sequence. The complete protocol is given as supplementary data file

\begin{tabular}{ll}
\hline 3D-DESS MRI parameters & Total \\
\hline Acquisition time & $12: 24 \mathrm{~min}: \mathrm{s}$ \\
FOV & $242 \times 242 \times 78 \mathrm{~mm}^{3}$ \\
Acq matrix & $320 \times 320 \times 78$ \\
Acq voxel & $0.75 \times 0.75 \times 0.75 \mathrm{~mm}^{3}$ \\
Number of signal averages & 1 \\
TR & $11.2 \mathrm{~ms}$ \\
TE1 & $4.2 \mathrm{~ms}$ \\
TE2 & $7.7 \mathrm{~ms}$ \\
WFS (pix)/bandwidth $(\mathrm{Hz})$ & $1 / 355$ \\
Fat suppression & Selective water excitation \\
Parallel acquisition & No \\
\hline
\end{tabular}


quantitative and qualitative analysis. Qualitative analysis has been assessed using a 5-point Likert rating scale for overall technical image quality and a qualitative 4-point Likert scale for grading the artifacts. In addition, nerve continuity analysis and the focal detectability of the six intermediate points were evaluated (see Fig. 1 demonstrating nerve anatomy and intermediate points). As part of the quantitative analysis, the horizontal and vertical distance between $\mathrm{LN}$ and lingual cortical plate and the $\mathrm{LN}$ diameter at two defined locations were measured.

\section{Qualitative readout}

First, the overall image quality was evaluated using a modified 5-point Likert scale, where motion artifacts, pulsation, and ghosting in the molar region were considered [22]: 0, excellent image quality with full diagnostic interpretability of the LN and surrounding tissue; 1, good image quality with full diagnostic interpretability of the LN and surrounding tissue; 2 , acceptable image quality and diagnostic interpretability; 3, markedly reduced image quality and impaired diagnostic interpretability of the LN and tissue components; 4 , severely reduced image quality, allowing no diagnostic interpretability of LN and/or surrounding tissue. Second, artifacts in the region of the first, second, and third molars were graded by a 4-point Likert scale: 0 , absence of artifacts caused by dental restorations (none); 1 , minor artifacts caused by dental restorations (low); 2 , moderate artifacts caused by dental restorations (moderate); 3 , massive artifacts caused by dental restorations (high).

The nerve continuity analysis was done according to Fujii et al. [17] by dividing the nerve into a proximal (from IP1 to IP3) and distal part (from IP3 to IP6) and checked for their continuous visibility using a modified 5 -point scale: 4 , excellent $=$ both the proximal and distal portions of the nerve identified; 3 , good = both the proximal and distal portions of the nerve identified but not continuous, with the distinction whether the interruption was proximal or distal; 2 , fair = only the proximal portion of the nerve identified; 1 , poor $=$ only the proximal portion of the nerve identified but not continuous; 0 , none $=$ the nerve not identified (Fig. 1).

Additionally, the LN was checked for the focal detectability of six intermediate points, which were selected in the extracranial course of the trigeminal nerve with special focus on the mandibular division (V3): the first point was the oval foramen (IP1), where the mandibular nerve (V3) descends and splits up in an anterior and posterior branch. The second intermediate point is localized at the point where the mandibular nerve branches into its various branches with a focus on the branching of the inferior alveolar nerve (IAN) and the LN (IP2). The LN, part of the posterior part, lying medial and in front of the IAN and beneath the lateral pterygoid muscle (LPM), is joined by the chorda tympani, a branch of the facial nerve (VII), and runs towards the medial pterygoid muscle (MPM). The nerve runs between the MPM and the ramus of the mandibula and crosses towards the side of the tongue. The point where the LN starts running from laterally to medially (IP3), the one and only intermediate point for the LN selected by Fujii et al., is the third intermediate point. The $\mathrm{LN}$ crosses the duct of the submandibular gland and runs in the third molar region where the fourth intermediate point was selected at the level of the third molar whether present or absent (IP4). The LN, characterized by its large anatomical variability, partially gives off a branch to
Fig. 1 Photorealistic 3-dimensional (3D) visualization of a study participant's cone beam computed tomography (CBCT) using cinematic rendering (CR). The division of the mandibular and lingual nerve (LN) into a proximal (IP1-IP3) and distal portion (IP3-IP6) with the 6 intermediate points (IP) is visualized. IP1, oval foramen; IP2, branching into the IAN and LN; IP3, LN starts running from laterally to medially; IP4, LN at the level of the third molar; IP5, gingival branch of the LN; IP6, $\mathrm{LN}$ at the height of the second molar

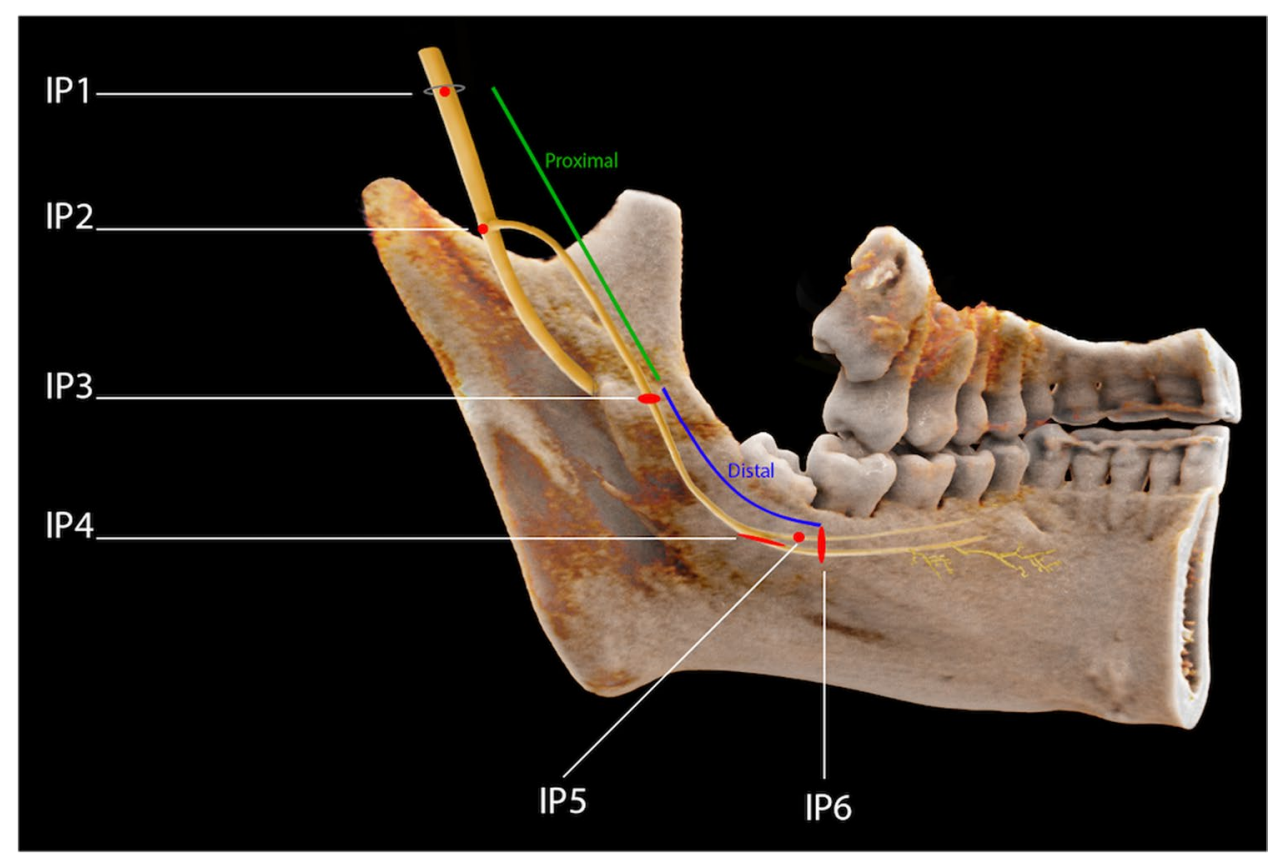


the lingual gingiva that is referred to in the literature as "the gingival branch of the lingual nerve" [23] or the "collateral nerve twigs"(IP5) [24]. The last and sixth intermediate point is at the height of the tangent through the distal margin of the crown of the second molar (IP6) (Figs. 1 and 2).

The six intermediate points defined over the LN course were selected with special respect to the anatomical areas that are particularly vulnerable for the $\mathrm{LN}$ during third molar extraction treatments. Their detectability was checked using the following 4-point scale: 4 , yes; 3 , probably yes; 2 , probably no; 1 no.

\section{Quantitative readout}

The information about the horizontal and vertical distance between the LN and the alveolar ridge of the lingual cortical plate is important for surgical interventions on the third molars and was determined using axial and coronal images of the 3D-DESS sequence.

According to Burian et al. in 2019, the LN diameter was measured at a proximal and distal location to examine the preoperative nerve diameter assessment. The proximal measurement took place $2 \mathrm{~cm}$ caudal to the oval foramen in the infratemporal fossa and the distal measurement point was at the entrance of the $\mathrm{LN}$ into the pterygomandibular space opposite to the third molar region [18].

\section{Statistical analyses}

Statistical analyses were performed using IBM SPSS Statistics software (version 25.0, IBM Corp. Armonk, NY, USA). The average of the nerve continuity analysis scores and intermediate point detectability values of each reader was calculated. In addition, the average scores of all three readers were calculated. A two-way mixed intraclass correlation
Fig. 2 a Coronal and b-d axial reconstruction of the $3 \mathrm{D}$-DESS sequence showing the intermediate points (IP). IP1 (a, short arrow): mandibular nerve within the oval foramen; IP2 (a, long arrow): branching of the inferior alveolar nerve (IAN) and the lingual nerve (LN). In the axial images, the IAN (b, short arrow) and the point in the course of the LN (b, long arrow) where it starts running from laterally to medially (IP3) are visible. IP4 (c, short arrow): LN at the third molar level; IP5 (c, long arrow): LN's gingival branch. IP6 (d, long arrow) represents the $\mathrm{LN}$ at the second molar level and its further insertion into the tongue (d, short arrow)
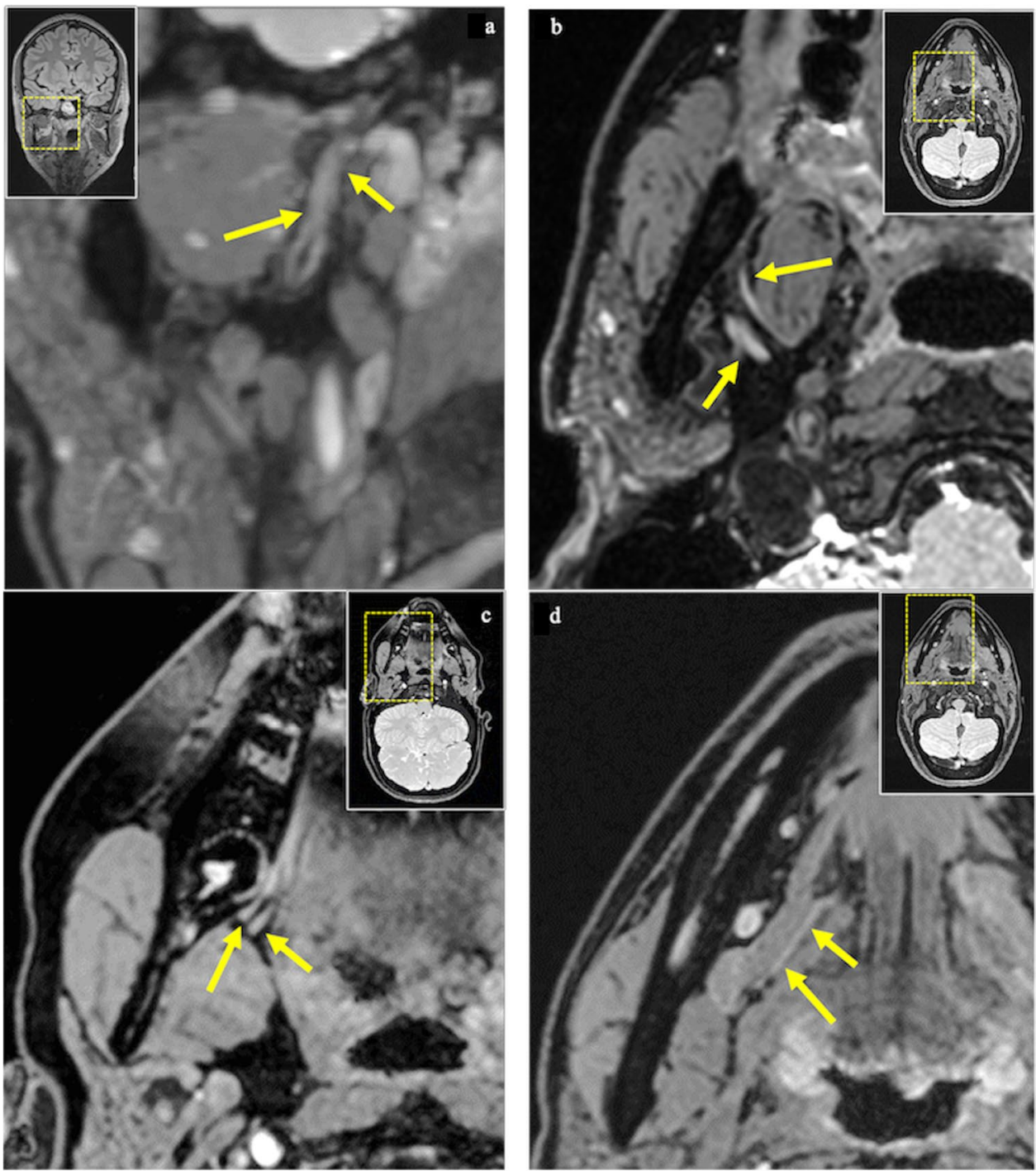
coefficient (ICC) with a two-sided $95 \%$ confidence interval (CI) was used to calculate the absolute agreement of the intra- and interobserver variability of the nerve continuity analysis. The strength of agreement beyond chance obtained can be interpreted as follows: poor, $<0.5$; moderate, 0.5-0.75; good, 0.75-0.9; excellent, $>0.9$ [25]. The power analysis was estimated using existing literature [17]. Average score and inter- and intra-reader agreements regarding the qualitative assessment of image quality and measurements of artifacts were also analyzed. Regarding the six intermediate points' focal detectability, intra- and interobserver agreement was reported as a percentage agreement. The nonparametric Mann-Whitney $U$ test based on a two-tailed test with a significance of $p<0.05$ was chosen to check whether there are significant differences between the diameter of the LN and the distance between the LN and the alveolar crest of the lingual cortical plate on the left and right side.

\section{Results}

\section{Inter-reader and intra-reader agreement}

The inter-reader and the intra-reader agreement between readers $\mathrm{A}, \mathrm{B}$, and $\mathrm{C}$ was moderate or good regarding the qualitative overall technical image quality (inter-reader ICC $=0.667$, intra-reader ICC $=0.764$; both $p<0.001)$ and the artifact severity (inter-reader ICC $=0.698$, intra-reader ICC $=0.721$; both $p<0.001$ ).

The inter-reader and intra-reader agreement between the three readers regarding the nerve continuity analysis was good (inter-reader ICC $=0.764$, intra-reader ICC $=0.749$; both $p<0.001)$. Regarding the average percentage agreement in all intermediate points, the interreader agreement was $77 \%$ and the intra-reader agreement was $87 \%$ (Table 3 ).

\section{Qualitative results}

The average score of all readers for the overall image quality was $0.382 \pm 0.7$. Thus, the image quality was excellent with full diagnostic interpretability of the LN and surrounding tissue.

Artifacts caused by dental restoration in the molar region showed an average evaluation score of $0.256 \pm 0.5$. Thus, it can be stated that the DESS images showed ordinarily absence of artifacts caused by dental restorations.

The nerve continuity analysis demonstrated an average score of $3.3 \pm 0.46$ (good). In $35 \%$ of the cases, the entire course of the LN was fully continuously visible at least from IP1 to IP6, and in most cases until its insertion into the tongue. In $10 \%$ of the cases, the proximal part of the LN was not entirely continuously visible; in all cases, the interruption took place at IP2. In $60 \%$ of the cases, the distal part of the nerve was not entirely continuously visible; in most of these cases, the interruption was in the section where the LN runs forward to the distal margin of the second molar (Table 4).

The average detectability score of all intermediate points was $3.7 \pm 0.41$. From IP1 to IP4, the detectability was excellent; IP5 had a slightly lower average score ( $3.5 \pm 0.74)$ but could still be considered highly visible. In contrast to the first five intermediate points, IP6 was least detectable with an average score of $2.79 \pm 1.05$ (Table 4).

the absolute agreement using intraclass correlation coefficients (ICC) with a two-sided $95 \%$ confidence interval (CI) was assessed
Table 3 The intra- and interobserver variability of the 3 readers (A, B, and C). For the IP detectability analysis, the agreement was expressed as a percentage, while for the nerve continuity analysis,

\begin{tabular}{|c|c|c|c|c|c|c|c|}
\hline Lingual nerve & $\mathrm{A}$ and $\mathrm{B}$ & $\mathrm{B}$ and $\mathrm{C}$ & $\mathrm{C}$ and $\mathrm{A}$ & $\mathrm{A} 1$ and $\mathrm{A} 2$ & $\mathrm{~B} 1$ and $\mathrm{B} 2$ & $\mathrm{C} 1$ and $\mathrm{C} 2$ & Average \\
\hline $\begin{array}{l}\text { Intermediate } \\
\text { point } 1\end{array}$ & $94.7 \%$ & $94.7 \%$ & $100 \%$ & $100 \%$ & $94.7 \%$ & $100 \%$ & $97.4 \%$ \\
\hline $\begin{array}{l}\text { Intermediate } \\
\text { point } 2\end{array}$ & $92.1 \%$ & $86.8 \%$ & $89.5 \%$ & $97.3 \%$ & $94.7 \%$ & $94.7 \%$ & $92.5 \%$ \\
\hline $\begin{array}{l}\text { Intermediate } \\
\text { point } 3\end{array}$ & $100 \%$ & $100 \%$ & $100 \%$ & $100 \%$ & $100 \%$ & $100 \%$ & $100 \%$ \\
\hline $\begin{array}{l}\text { Intermediate } \\
\text { point } 4\end{array}$ & $78.9 \%$ & $89.5 \%$ & $86.8 \%$ & $92.1 \%$ & $86.8 \%$ & $92.1 \%$ & $87.7 \%$ \\
\hline $\begin{array}{l}\text { Intermediate } \\
\text { point } 5\end{array}$ & $52.6 \%$ & $73.7 \%$ & $55.3 \%$ & $78.9 \%$ & $84.2 \%$ & $73.7 \%$ & $69.7 \%$ \\
\hline $\begin{array}{l}\text { Intermediate } \\
\text { point } 6\end{array}$ & $28.9 \%$ & $34.2 \%$ & $36.8 \%$ & $71.1 \%$ & $68.4 \%$ & $39.5 \%$ & $46.5 \%$ \\
\hline $\begin{array}{l}\text { Nerve Continu- } \\
\text { ity Score (ICC } \\
(95 \% \text { CI) })\end{array}$ & $0.661(0.44-0.81)$ & $0.887(0.79-0.94)$ & $0.744(0.61-0.88)$ & $0.634(0.38-0.8)$ & $0.722(0.53-0.85)$ & $0.89(0.79-0.94)$ & 0.756 \\
\hline
\end{tabular}


Table 4 Average scores of the 3 readers (A, B, and C, 1 first readout and $2 \mathrm{~s}$ readout) evaluating the lingual nerve continuity by using a 5 -point scale $(4=$ excellent; $3=$ good; $2=$ fair; $1=$ poor; $0=$ none $)$ and the detectability of the intermediate points over the entire course of the lingual nerve by using a 4 -point scale $(4=$ yes; $3=$ probably yes; $2=$ probably no; $1=$ no)

\begin{tabular}{|c|c|c|c|c|c|c|c|}
\hline Lingual nerve & Reader A1 & Reader B1 & Reader C1 & Reader A2 & Reader B2 & Reader C2 & Average \\
\hline ntermediate point 1 & SD 0) & 95 (SD 0.23) & $4(\mathrm{SD}$ & 4( & 0) & & 3.99 \\
\hline Intermediate point 2 & $3.95(\mathrm{SD} 0.23)$ & 3.92 (SD 0.27) & $3.95(\mathrm{SD} 0.23)$ & $3.92(\mathrm{SD} 0.36)$ & $3.95(\mathrm{SD} 0.23)$ & 3.95 (SD 0.24) & 3.94 (SD 0.26 \\
\hline Intermediate point 3 & $4(\mathrm{SD} 0)$ & $4(\mathrm{SD} 0)$ & 4 (SD 0) & 4 (SD 0) & $4(\mathrm{SD} 0)$ & 4 (SD 0) & 4 (SD 0) \\
\hline Intermediate point 4 & 3.79 (SD 0 & $3.95(\mathrm{SD} 0.23)$ & $3.95(\mathrm{SD} 0.23)$ & 3.76 (SD 0.59) & $3.82(\mathrm{SD} 0.46)$ & 3.97 (SD 0.16) & $3.87(\mathrm{SD} 0.37)$ \\
\hline Intermediate point 5 & 3.07 (SD 1) & $3.74(\mathrm{SD} 0.50)$ & 3.71 (SD 0.77) & 3.15 (SD 0.86) & 3.68 (SD 0.53) & 3.66 (SD 0.78) & $3.5(\mathrm{SD} 0.74)$ \\
\hline Intermediate point 6 & 2.45 (SD 1.33) & $3.02(\mathrm{SD} 0.79)$ & 3.05 (SD 1.04) & $2.47(\mathrm{SD} 1.31)$ & $3.05(\mathrm{SD} 0.8)$ & $2.71(\mathrm{SD} 1.01)$ & $2.79($ SD 1.05$)$ \\
\hline Nerve Continuity Score & 3.37 (SD 0.49) & 3.34 (SD 0.48) & $3.32(\mathrm{SD} 0.47)$ & $3.3(\mathrm{SD} 0.41)$ & 3.26 (SD 0.45) & 3.34 (SD 0.48) & $3.32(\mathrm{SD} 0.46)$ \\
\hline
\end{tabular}

\section{Quantitative results}

The mean horizontal distance between the $\mathrm{LN}$ and the alveolar crest of the lingual cortical plate was $1.05 \pm 1.0 \mathrm{~mm}$, and the mean vertical distance was $4.65 \pm 1.2 \mathrm{~mm}$. No significant differences were registered between both sides (horizontal right vs. left $p=0.435$, vertical right vs. left $p=0.311$ ) (Fig. 3; Table 5).

The mean nerve diameter of the $\mathrm{LN} 2 \mathrm{~cm}$ caudal to the oval foramen was $1.42 \pm 0.2 \mathrm{~mm}$. The mean diameter at the entrance of the LN into the pterygomandibular space opposite to the third molar region was $1.06 \pm 0.2 \mathrm{~mm}$. No significant differences between the left and right sides were registered (proximal right vs. left $p=0.454$, distal right vs. left $p=0.402)$ (Fig. 3; Table 6).

\section{Discussion}

This study investigated the visualization of the extracranial course of the LN with special respect to the anatomical relationship of the third molar teeth by using a 3D-DESS MRI sequence. This study's results showed the feasibility of the focal and continuous detectability of the course of the LN with a high reproducibility. It was possible to generate reliable measurements of the nerve diameter and of the horizontal and vertical distance from the LN to the lingual cortical plate with a high concordance of both sides, which were consistent with the results of previous radiographic studies and anatomical cadaver studies [18, 26-28].

Despite challenges in detecting the extracranial peripheral nerves in clinical routine, several studies using different imaging modalities showed that direct visualization is feasible and accurate $[14,15,17,18]$. Previous studies also proved that direct MRI visualization of the $\mathrm{LN}$ using the 3D-DESS sequence is possible [17, 18]. This sequence, currently successfully applied in musculoskeletal imaging and parotid tumor and facial nerve localization, with its fatsuppression technique, is well suited for depicting the LN due to the simultaneous application of the water excitation technique, providing uniform fat suppression and allowing the clear visualization of the LN independent of the surrounding fatty tissue [20].

In this study, it was possible to continuously visualize the entire $\mathrm{LN}$ course from the oval foramen to the distal margin of the second molar. Despite the small size, the ramification of the nerve, and the proximity to other anatomical structures, in some cases, it was possible to visualize the nerve until its insertion into the tongue. In almost all cases (92\%), it was possible to display the LN continuously at the level of the third molar; meanwhile, the continuous visibility got lost in $60 \%$ of the cases in the area where the LN runs forward to the second molar and further. The fact of not being able to display the LN at this level of its course could be explained by various causes, such as the anatomy of the nerve, the imaging technique, or the readers. The achieved inter-reader agreement showed that despite the great differences in the readers' experience, this diagnostic method is suitable with the potential to be established in clinical routine in the future. However, prospective studies are required to assess the therapeutic and clinical impact in patients undergoing third molar removal having preoperative MRI.

Regarding the quantitative parameters, we got reproducible nerve diameter measurements for the proximal and distal location showing no significant differences between both sides. Burian et al.'s MRI study presented slightly higher proximal and distal median (proximal $+0.2 \mathrm{~mm}$, distal $+0.3 \mathrm{~mm}$ ) values than this study; regarding the upper and lower range proximal and distal, the values obtained showed high concordance [18]. Considering that no significant differences were found in diameter inter-side, these values can be seen as comparable, as minimal differences may have occurred due to anatomical variation or to the resolution of the DESS images. The mean horizontal distance from the LN to the lingual cortical plate and lingual crest of the mandible using MRI visualization was $1.05 \pm 1.0 \mathrm{~mm}$ and the mean vertical distance was $4.65 \pm 1.2 \mathrm{~mm}$. These parameters important for performing third molar extraction 
Fig. 3 a, b Box plots of the lingual nerve diameter in proximal and distal locations (proximal right vs. left $p=0.454$, distal right vs. left $p=0.402$ ) and of the horizontal and vertical distances (horizontal right vs. left $p=0.435$, vertical right vs. left $p=0.311$ ) from the lingual nerve to the lingual cortical plate for the left and right side (Mann-Whitney $U$ test $p<0.05)$. IQR, interquartile range; Q1, first quartile; Q3, third quartile. •: IQR (Q3-Q1), —: median
Lingual Nerve Diameter

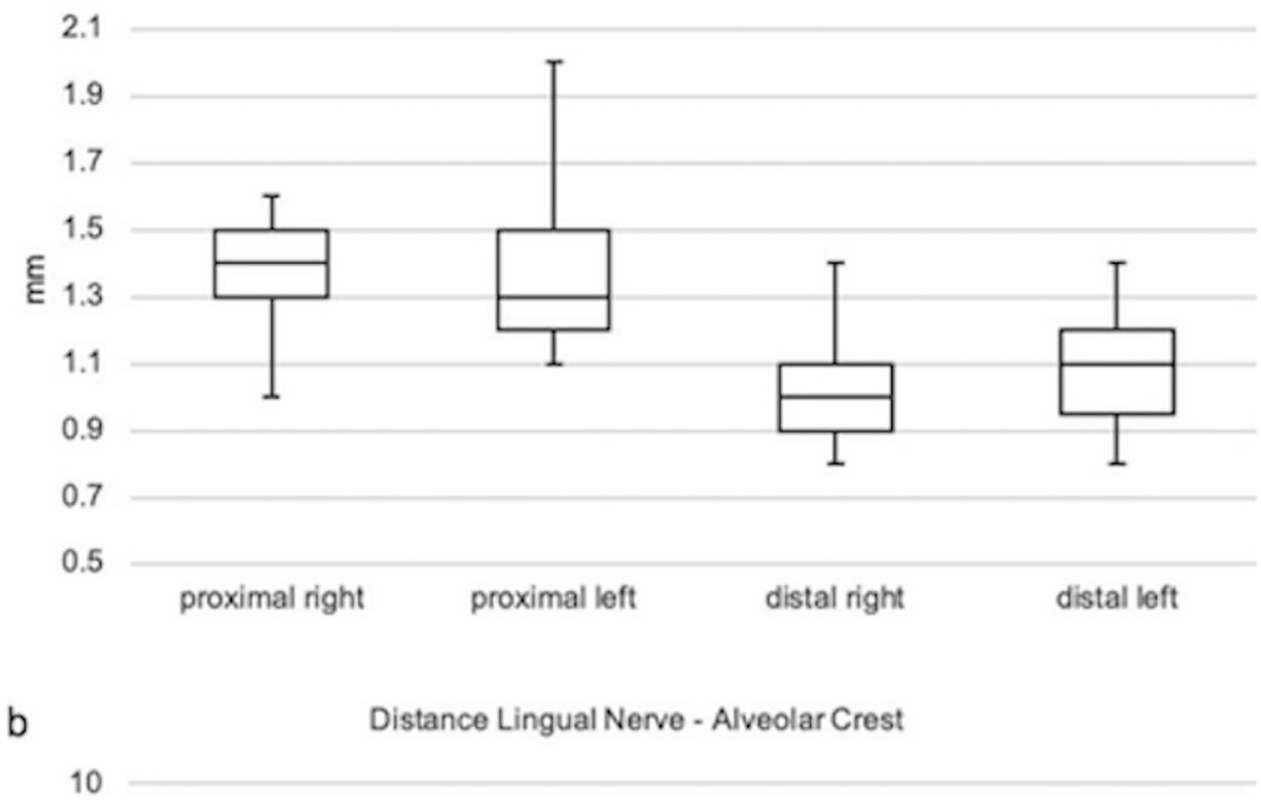

8

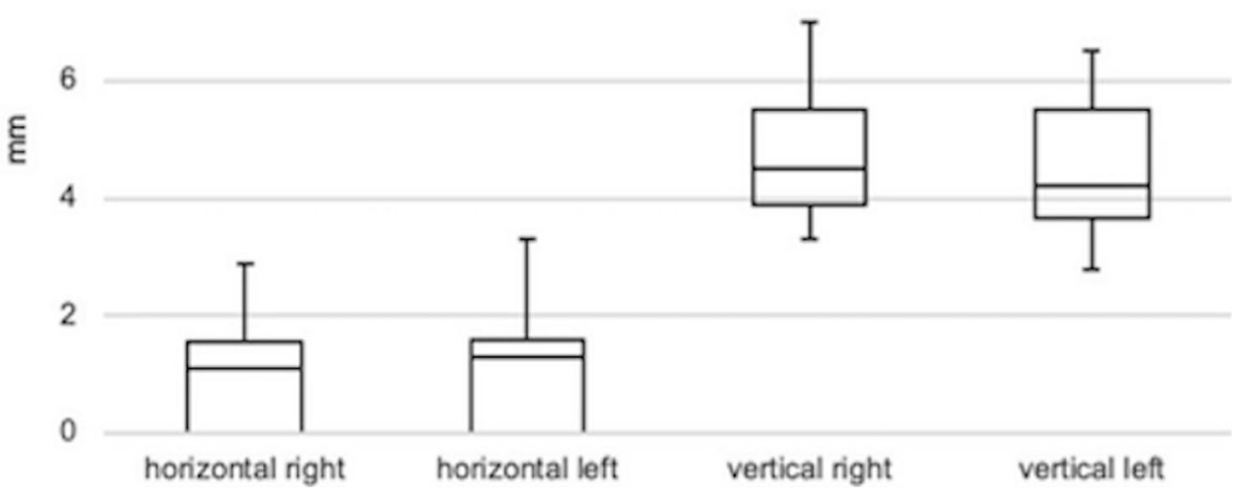

with minimal risk to LN damage were consistent with some previously performed anatomical cadaver studies [26-28]. Other studies demonstrated about 3 to $8 \mathrm{~mm}$ higher mean horizontal, and about 2 to $3 \mathrm{~mm}$ higher mean vertical distances [29, 30]. However, this is probably based on the high intervariation of the $\mathrm{LN}$ in the third molar region.

To date, the cost-effectiveness is not given yet, as dental MRI imaging is still not considered a standard diagnostic procedure in the context of preoperative radiological assessment prior to third molar extraction treatment. However, the constantly increasing number of MRI reports in the dental field confirms the importance and perspectives that are opening up due to the targeted use of this radiation-free imaging modality. Further refinement of this MRI protocol and possible application in MR neurography using high-field-strength MRI could allow the differentiation of the skull base from extracranial nerve segments in T2-weighted images with high soft tissue contrast and homogenous fat, arterial, and venous suppression. This accurate visualization might be useful in various other surgical procedures, such as dental implant placement, mandibular split osteotomy, or detection of post-traumatic trigeminal lesions [31, 32]. Therefore, MRI imaging might provide benefits for the patient and the clinician in challenging cases.

Several limitations in this study should be mentioned. Firstly, there is a methodical limitation because of the small cohort size. Therefore, it is challenging to make generally valid statements; larger cohorts and further investigations are needed to confirm these qualitative and quantitative measurements with higher certainty, allowing ideal sample size calculation. Secondly, the results of the image quality and artifact susceptibility evaluation should be interpreted with 
Table 5 The horizontal and vertical distance between the lingual nerve and lingual cortical plate was registered. No significant differences were found between the distances on the right side compared to the left side

\begin{tabular}{|c|c|c|c|}
\hline Lingual nerve $N=38$ & Right & Left & Average \\
\hline \multicolumn{4}{|c|}{ Alveolar crest - LN horizontal distance } \\
\hline Mean \pm standard deviation & $0.91(\mathrm{SD} \mathrm{1}) \mathrm{mm}$ & $1.18(\mathrm{SD} 1.1) \mathrm{mm}$ & 1.05 (SD 1) mm \\
\hline Median & $1.1 \mathrm{~mm}$ & $1.3 \mathrm{~mm}$ & $1.2 \mathrm{~mm}$ \\
\hline Lower range & $0 \mathrm{~mm}$ & $0 \mathrm{~mm}$ & $0 \mathrm{~mm}$ \\
\hline Upper range & $2.9 \mathrm{~mm}$ & $3.3 \mathrm{~mm}$ & $3.1 \mathrm{~mm}$ \\
\hline $\begin{array}{l}\text { Mann-Whitney } U \text {, significantly } \\
\text { different }(p<0.05) ?\end{array}$ & $\begin{array}{l}\text { Right versus left } \\
\text { No } \\
p \text { value }=0.435\end{array}$ & & \\
\hline \multicolumn{4}{|l|}{ Alveolar crest - LN vertical distance } \\
\hline Mean \pm standard deviation & $4.87(\mathrm{SD} 1.2) \mathrm{mm}$ & $4.42(\mathrm{SD} 1.3) \mathrm{mm}$ & $4.65(\mathrm{SD} 1.2) \mathrm{mm}$ \\
\hline Median & $4.6 \mathrm{~mm}$ & $4.2 \mathrm{~mm}$ & $4.4 \mathrm{~mm}$ \\
\hline Lower range & $3.3 \mathrm{~mm}$ & $2.8 \mathrm{~mm}$ & $3.1 \mathrm{~mm}$ \\
\hline Upper range & $7 \mathrm{~mm}$ & $6.5 \mathrm{~mm}$ & $6.8 \mathrm{~mm}$ \\
\hline $\begin{array}{l}\text { Mann-Whitney } U \text {, significantly } \\
\text { different }(p<0.05) ?\end{array}$ & $\begin{array}{l}\text { Right versus left } \\
\text { No } \\
p \text { value }=0.311\end{array}$ & & \\
\hline
\end{tabular}

Table 6 Mean, median, and lower and upper range of the lingual nerve diameter in a proximal and distal location for left and right side. No significant differences could be detected regarding the measurements of both sides

\begin{tabular}{lllllll}
\hline $\begin{array}{l}\text { Lingual nerve, } \\
N=38\end{array}$ & Proximal right & Proximal left & Average & Distal right & Distal left & Average \\
\hline Mean & $1.42($ SD 0.2) $\mathrm{mm}$ & $1.41(\mathrm{SD} 0.3) \mathrm{mm}$ & $1.42(\mathrm{SD} 0.2) \mathrm{mm}$ & $1.03(\mathrm{SD} 0.2) \mathrm{mm}$ & 1.08 (SD 0.2) mm & 1.06 (SD 0.2) mm \\
Median & $1.4 \mathrm{~mm}$ & $1.3 \mathrm{~mm}$ & $1.4 \mathrm{~mm}$ & $1 \mathrm{~mm}$ & $1.1 \mathrm{~mm}$ & $1.1 \mathrm{~mm}$ \\
Lower range & $1 \mathrm{~mm}$ & $1.1 \mathrm{~mm}$ & $1.1 \mathrm{~mm}$ & $0.8 \mathrm{~mm}$ & $0.8 \mathrm{~mm}$ & $0.8 \mathrm{~mm}$ \\
Upper range & $1.6 \mathrm{~mm}$ & $2 \mathrm{~mm}$ & $1.8 \mathrm{~mm}$ & $1.4 \mathrm{~mm}$ & $1.4 \mathrm{~mm}$ & $1.4 \mathrm{~mm}$ \\
Mann-Whitney $U$, & Proximal right versus left & & Distal right versus left & \\
significantly dif- & No & & & No & $p=0.402$ & \\
ferent $(p<0.05) ?$ & $p=0.454$ & & & & & \\
\hline
\end{tabular}

caution as adjacent implants or metallic restorations were considered as exclusion criteria. Nevertheless, the literature indicates various other sources of artifacts: stainless-steel brackets, NiTi archwires, zirconia reconstructions, fillings with glass ionomer cement, composite resins, or even the use of zinc phosphate-based cement can trigger artifacts in MRI, too. Therefore, to achieve standardized, reliable data, even though metallic artifacts were per design excluded, the influence of motion and other non-metallic artifacts sources could still be investigated here. Thirdly, there is the anatomical course of the $\mathrm{LN}$ with its variations, especially in the third molar region, which makes the evaluation more difficult. To be more precise, this concerns its relationship to the alveolar ridge of the lingual cortical plate, whether there is a gingival branch or not, the place where the looping around the submandibular duct takes place and the anatomic proximity to other structures and their arrangement. In addition, the small size of the nerve in the investigated area: the finer the structure, the more difficult it is to distinguish the nerve from the surrounding and partially overlapping anatomical structures. Despite this fact, it was possible to visualize the nerve until its insertion into the tongue. For better visualization, refinement of the sequence should be part of future investigations. Fourthly, most of the participants were young, so they hardly showed any artifacts caused by dental restorations. Future investigations should also examine the influence of these factors on the detectability of the LN. Therefore, the MRI sequence's refinement should also deal with the minimization or even elimination of this kind of artifacts, maybe using zero TE or ultra-short TE methods. Considering the MRI artifact susceptibility of the head and neck area, some features and modifications of the DESS sequence were beneficial for the optimization process in MRI data acquisition; e.g., the double-echo acquisition of the DESS sequence provides, in a simplified view, good T2 contrast from one echo and low artifacts from the other. Furthermore, the high contrast diminishes intravoxel dephasing artifacts due to magnetic field inhomogeneities. Finally, the omission of grappa acceleration reduced the noise at the center of the FOV and explained our DESS implementation's relatively long scan time. Nevertheless, future studies should focus on these issues-possibly using 
different readout trajectories- to further improve MRI of the oral cavity. In addition, further research could be conducted to optimize image quality with respect to the contrast to noise ratio of other tissues than the LN, as this appears to be relatively low for the evaluated DESS sequence.

Modern surgical management of third molar extractions aims to avoid lingual flap detachment to protect the LN in the retromolar space [9]. Therefore, detailed knowledge of the LN course, its diameter, and its distance to the lingual cortical plate in the third molar region potentially reduces the risk for the surgeon to a minimum in cases where the surgical area has to be extended into the lingual area, due to the position of the third molar. As with most studies available in the literature, this is an exploratory study testing the feasibility of nerve visualization. Based on these data confirming the visualization of the $\mathrm{LN}$ and further depicting its continuous course and considering the large heterogeneity in the literature regarding scan parameters and the use of different magnetic field strengths, further studies should be conducted, including randomized control trials examining the evidence of the individual MRI protocol in a given medical condition, to provide an evidence-based understanding of its use and propose further information on how preoperative radiological assessment might influence the clinical outcome. Although MRI imaging is not yet an integral part of everyday dentistry, a close positional relationship to adjacent anatomical structures, short MRI examination times, and high detectability for the entire continuous LN course with high concordance by readers with different training levels might justify the use of it. After being validated in larger cohort groups and pathological cases, the targeted use of this MRI sequence might have the potential to be integrated in clinical routine and thus avoid the unpleasant postoperative complications associated with LN damage for the patients.

\section{Conclusion}

The implemented MRI sequence allowed for mostly continuous visualization of the entire course of the lingual nerve from the oval foramen to the third molar region with high confidence and reproducibility. This enables a preoperative clarification of the LN position in patients undergoing surgical third molar extraction treatment. The use of this MRI technology generates reproducible information about the LN diameter and its horizontal and vertical distance to the lingual cortical plate. This information could prevent complications in various oral surgical procedures.

Supplementary Information The online version contains supplementary material available at https://doi.org/10.1007/s00784-021-04185-z.
Acknowledgements The authors thank Marcel Müller, Statistical Services, Center of Dental Medicine, University of Zurich, Zurich, for his assistance in the statistical analysis of the data.

Author contribution All authors contributed to the study conception and design. Data acquisition, analysis, and interpretation were performed by Adib Al-Haj Husain, Silvio Valdec, Bernd Stadlinger, Martin Rücker, Marco Piccirelli, and Sebastian Winklhofer. The first draft of the manuscript was written by Adib Al-Haj Husain and all authors commented on previous versions of the manuscript. All authors read and approved the final version of the manuscript.

Funding Open access funding provided by University of Zurich. This research received financial support by Dentsply Sirona (York, USA).

This study (BASEC-Nr. 2017-01053) was approved by the Cantonal Ethics Commission of Zurich (Switzerland). Additionally, this study was performed in accordance with the 1964 Helsinki Declaration and its later revised ethical standards.

\section{Declarations}

Conflict of interest The authors declare no competing interests.

Open Access This article is licensed under a Creative Commons Attribution 4.0 International License, which permits use, sharing, adaptation, distribution and reproduction in any medium or format, as long as you give appropriate credit to the original author(s) and the source, provide a link to the Creative Commons licence, and indicate if changes were made. The images or other third party material in this article are included in the article's Creative Commons licence, unless indicated otherwise in a credit line to the material. If material is not included in the article's Creative Commons licence and your intended use is not permitted by statutory regulation or exceeds the permitted use, you will need to obtain permission directly from the copyright holder. To view a copy of this licence, visit http://creativecommons.org/licenses/by/4.0/.

\section{References}

1. Berberi A, Le Breton G, Mani J, Woimant H, Nasseh I (1993) Lingual paresthesia following surgical placement of implants: report of a case. Int J Oral Maxillofac Implants 8:580-582

2. Sandstedt P, Sörensen S (1995) Neurosensory disturbances of the trigeminal nerve: a long-term follow-up of traumatic injuries. J Oral Maxillofac Surg 53:498-505

3. Valmaseda-Castellón E, Berini-Aytés L, Gay-Escoda C (2000) Lingual nerve damage after third lower molar surgical extraction. Oral Surg Oral Med Oral Pathol Oral Radiol Endod 90:567-573

4. Fielding AF, Rachiele DP, Frazier G (1997) Lingual nerve paresthesia following third molar surgery: a retrospective clinical study. Oral Surg Oral Med Oral Pathol Oral Radiol Endod 84:345-348

5. Lehman CD, Bartoshuk LM, Catalanotto FC, Kveton JF, Lowlicht RA (1995) Effect of anesthesia of the chorda tympani nerve on taste perception in humans. Physiol Behav 57:943-951

6. Shafer DM, Frank ME, Gent JF, Fischer ME (1999) Gustatory function after third molar extraction. Oral Surg Oral Med Oral Pathol Oral Radiol Endod 87:419-428

7. Lopes V, Mumenya R, Feinmann C, Harris M (1995) Third molar surgery: an audit of the indications for surgery, postoperative complaints and patient satisfaction. Br J Oral Maxillofac Surg 33:33-35 
8. Renton T, McGurk M (2001) Evaluation of factors predictive of lingual nerve injury in third molar surgery. Br J Oral Maxillofac Surg 39:423-428

9. Pippi R, Spota A, Santoro M (2017) Prevention of lingual nerve injury in third molar surgery: literature review. J Oral Maxillofac Surg 75:890-900

10. Coulthard P, Kushnerev E, Yates JM, Walsh T, Patel N, Bailey E et al (2014) Interventions for iatrogenic inferior alveolar and lingual nerve injury. Cochrane Database Syst Rev 16:CD005293

11. Dula K, Bornstein MM, Buser D, Dagassan-Berndt D, Ettlin DA, Filippi A et al (2014) SADMFR guidelines for the use of cone-beam computed tomography/ digital volume tomography. Swiss Dent J 124:1169-1183

12. Chhabra A, Zhao L, Carrino JA, Trueblood E, Koceski S, Shteriev F et al (2013) MR neurography: advances. Radiol Res Pract 2013:809568

13. Naraghi AM, Awdeh H, Wadhwa V, Andreisek G, Chhabra A (2015) Diffusion tensor imaging of peripheral nerves. Semin Musculoskelet Radiol 19:191-200

14. Benninger B, Kloenne J, Horn JL (2013) Clinical anatomy of the lingual nerve and identification with ultrasonography. Br J Oral Maxillofac Surg 51:541-544

15. Manoliu A, Ho M, Nanz D, Dappa E, Boss A, Grodzki DM et al (2016) MR neurographic orthopantomogram: ultrashort echo-time imaging of mandibular bone and teeth complemented with highresolution morphological and functional MR neurography. J Magn Reson Imaging 44:393-400

16. Wu W, Wu F, Liu D, Zheng C, Kong X, Shu S et al (2020) Visualization of the morphology and pathology of the peripheral branches of the cranial nerves using three-dimensional highresolution high-contrast magnetic resonance neurography. Eur J Radiol 132:109137

17. Fujii H, Fujita A, Yang A, Kanazawa H, Buch K, Sakai O et al (2015) Visualization of the peripheral branches of the mandibular division of the trigeminal nerve on 3D double-echo steadystate with water excitation sequence. AJNR Am J Neuroradiol 36:1333-1337

18. Burian E, Probst FA, Weidlich D, Cornelius CP, Maier L, Robl $\mathrm{T}$ et al (2019) MRI of the inferior alveolar nerve and lingual nerve-anatomical variation and morphometric benchmark values of nerve diameters in healthy subjects. Clin Oral Investig $24: 2625-2634$

19. Al-Haj Husain A, Stadlinger B, Winklhofer S, Müller M, Piccirelli M, Valdec S (2021) Mandibular third molar surgery: intraosseous localization of the inferior alveolar nerve using 3D double-echo steady-state MRI (3D-DESS). Diagnostics (Basel) 11:1245

20. Qin Y, Zhang J, Li P, Wang Y (2011) 3D double-echo steady-state with water excitation MR imaging of the intraparotid facial nerve at 1.5T: a pilot study. AJNR Am J Neuroradiol 32:1167-1172

21. Schneider T, Filo K, Kruse AL, Locher M, Grätz KW, Lübbers HT (2014) Variations in the anatomical positioning of impacted mandibular wisdom teeth and their practical implications. Swiss Dent J 124:520-538

22. Guggenberger R, Winklhofer S, Osterhoff G, Wanner GA, Fortunati M, Andreisek G et al (2012) Metallic artefact reduction with monoenergetic dual-energy CT: systematic ex vivo evaluation of posterior spinal fusion implants from various vendors and different spine levels. Eur Radiol 22:2357-2364

23. Kocabiyik N, Varol A, Sencimen M, Ozan H (2009) An unnamed branch of the lingual nerve: gingival branch. Br J Oral Maxillofac Surg 47:214-217

24. Kim SY, Hu KS, Chung IH, Lee EW, Kim HJ (2004) Topographic anatomy of the lingual nerve and variations in communication pattern of the mandibular nerve branches. Surg Radiol Anat 26:128-135

25. Koo TK, Li MY (2016) A guideline of selecting and reporting intraclass correlation coefficients for reliability research. J Chiropr Med 15:155-163

26. Hölzle FW, Wolff KD (2001) Anatomic position of the lingual nerve in the mandibular third molar region with special consideration of an atrophied mandibular crest: an anatomical study. Int J Oral Maxillofac Surg 30:333-338

27. Kiesselbach JE, Chamberlain JG (1984) Clinical and anatomic observations on the relationship of the lingual nerve to the mandibular third molar region. J Oral Maxillofac Surg 42:565-567

28. Dias GJ, de Silva RK, Shah T, Sim E, Song N, Colombage S et al (2015) Multivariate assessment of site of lingual nerve. Br J Oral Maxillofac Surg 53:347-351

29. Erdogmus S, Govsa F, Celik S (2008) Anatomic position of the lingual nerve in the mandibular third molar region as potential risk factors for nerve palsy. J Craniofac Surg 19:264-270

30. Pogrel MA, Renaut A, Schmidt B, Ammar A (1995) The relationship of the lingual nerve to the mandibular third molar region: an anatomic study. J Oral Maxillofac Surg 53:1178-1181

31. Van der Cruyssen F, Peeters F, Croonenborghs TM, Fransen J, Renton T, Politis C et al (2021) A systematic review on diagnostic test accuracy of magnetic resonance neurography versus clinical neurosensory assessment for post-traumatic trigeminal neuropathy in patients reporting neurosensory disturbance. Dentomaxillofac Radiol 50(1):20200103

32. Van der Cruyssen F, Croonenborghs TM, Renton T, Hermans R, Politis C, Jacobs R et al (2021) Magnetic resonance neurography of the head and neck: state of the art, anatomy, pathology and future perspectives. Br J Radiol 94(1119):20200798

Publisher's Note Springer Nature remains neutral with regard to jurisdictional claims in published maps and institutional affiliations. 